\title{
Dielectric and impedance properties of sintered magnesium aluminum silicate glass-ceramic
}

\author{
Abhijit PRASAD ${ }^{*}$, Amitabha BASU \\ Department of Applied Physics, Indian School of Mines, Dhanbad 826004, India \\ Received: September 18, 2012; Revised: February 05, 2013; Accepted: February 06, 2013 \\ CThe Author(s) 2013. This article is published with open access at Springerlink.com
}

\begin{abstract}
Magnesium aluminum silicate $\left(\mathrm{MgO}-\mathrm{Al}_{2} \mathrm{O}_{3}-\mathrm{SiO}_{2}, \mathrm{MAS}\right)$ glass-ceramic was prepared via sintering route. X-ray diffraction (XRD) analysis indicates the formation of multiphase compound with magnesium silicate fluoride and cordierite as major phases. Dielectric studies of the compound shows a strong frequency dispersion of permittivity in the low frequency region followed by a nearly frequency-independent behavior in the high frequency region. The presence of alkali ions $\left(\mathrm{K}^{+}\right)$in MAS glass-ceramic results in higher dielectric constant and higher losses. Dielectric loss $(\tan \delta)$ seems to be reduced at higher frequencies after reaching the instrumental saturation at $1 \mathrm{kHz}$. Electrical properties of the material have been studied using a complex impedance spectroscopy (CIS) technique in a wide temperature $\left(30-500{ }^{\circ} \mathrm{C}\right)$ and frequency $(100 \mathrm{~Hz}-5 \mathrm{MHz})$ ranges. The complex impedance plots reveal the contribution of bulk as well as grain boundary effects in it. The bulk resistance, evaluated from complex impedance spectrum, has been observed to decrease with the rise in temperature, showing a typical negative temperature coefficient of resistance (NTCR) behavior. The smaller activation energy $\left(E_{\mathrm{a}}\right)$ of the compound within moderate temperature range suggests the presence of ions $\left(\mathrm{K}^{+}\right.$and $\left.\mathrm{F}^{-}\right)$and singly ionized oxygen vacancies in the conduction process. The nature of variation of the electrical conductivity, and the values of activation energy of different temperature regions, suggest that the conduction process is of mixed type.
\end{abstract}

Keywords: glass-ceramic; conductivity; dielectric properties; electrical properties

\section{Introduction}

Magnesium aluminum silicate $\left(\mathrm{MgO}-\mathrm{Al}_{2} \mathrm{O}_{3}-\mathrm{SiO}_{2}\right.$, MAS) glass-ceramic systems belong to a special class of technologically important advanced ceramics, having applications in the areas related to ultrahigh vacuum, high temperature, high voltage, etc. They have high thermal stability, low thermal conductivity, good mechanical strength and very high electrical insulation and are also vacuum compatible [1-6]. The

* Corresponding author.

E-mail: abhijit_133@rediffmail.com properties of MAS glass-ceramic such as hardness, machinability, conductivity and other properties depend on the composition and microstructure.

Complex impedance spectroscopy (CIS) is a well-known and powerful technique for investigating polycrystalline materials, where the contribution of different processes, such as bulk conduction, grain boundary conduction and transport across electrode-sample interface, can be resolved in the frequency domain. The results can be often correlated with the properties such as composition, microstructure, defects and dielectric properties of the sample. This technique has the advantage over DC techniques of being able to separate the electrical responses (i.e., 
transport properties) of different regions of a material (i.e., the resistance of the grain boundaries and that of grains can be easily separated in most of the polycrystalline materials), provided their electrical responses are within the measuring range of the instrumentation. In this paper, we present a systematic study of MAS glass-ceramic prepared by sintering route and the influence of temperature and frequency on dielectric and impedance properties with a view of understanding the conduction mechanism involved.

\section{Experimental procedures}

MAS glass-ceramic sample was prepared via sintering route, using three-stage heating schedule involving calcination, nucleation and crystallization processes. The initial charge was prepared by taking analytical reagent (AR) grade chemicals in proper weight percentages of $\mathrm{SiO}_{2} 47.5 \%, \mathrm{Al}_{2} \mathrm{O}_{3} 16.5 \%, \mathrm{MgO} 18.5 \%$, $\mathrm{K}_{2} \mathrm{O} 9.5 \%, \mathrm{~B}_{2} \mathrm{O}_{3} 8 \%$. In the first stage, the initial charge was thoroughly mixed in agate mortar for $2 \mathrm{~h}$, including wet mixing in acetone media for $1 \mathrm{~h}$. The mixture was then calcined at $950{ }^{\circ} \mathrm{C}$ for $6 \mathrm{~h} .7 \mathrm{wt} \%$ $\mathrm{MgF}_{2}$ was added to the calcined charge and was pulverized using agate mortar and pestle for $2 \mathrm{~h}$. Finely milled charge was seasoned in $5 \% \mathrm{H}_{3} \mathrm{PO}_{4}$ acid solution in acetone medium for a period up to $72 \mathrm{~h}$. Cylindrical pellets of $12 \mathrm{~mm}$ diameter and $3 \mathrm{~mm}$ thickness were prepared in a hydraulic press using $80 \mathrm{MPa}$ pressure. They were then sintered using a two-step heating program. In the first step, the compact was heated up to $670-700{ }^{\circ} \mathrm{C}$ for $2-4 \mathrm{~h}$ to ensure good nucleation and initiate crystal growth. These pellets were then sintered with optimized temperature and time $\left(1000{ }^{\circ} \mathrm{C}, 3 \mathrm{~h}\right)$ in air atmosphere. Approximately $15 \mathrm{mg}$ of crushed fine powders of the sintered pellets were used for differential thermal analysis (Model Pyris Diamond TG-DTA), which was heated up to $905{ }^{\circ} \mathrm{C}$ at the rate of $10{ }^{\circ} \mathrm{C} / \mathrm{min}$ in $\mathrm{O}_{2}$ atmosphere using alumina as reference. The sintered sample was crushed and the phase constitution was studied by X-ray diffraction (XRD) using Philips Xpert-pro X-ray Powder Diffractometer in a wide range of Bragg angles $2 \theta$ $\left(10^{\circ} \leqslant 2 \theta \leqslant 70^{\circ}\right)$ being irradiated by $\mathrm{Cu} \mathrm{K} \alpha$ $(\lambda=1.5405 \AA)$. The bulk density for the sintered sample was measured by Archimedes method (i.e., immersion in distilled water). Scanning electron microscopy (SEM) was used to study the surface morphology of the ceramic. For dielectric measurements, the sintered pellets were polished with zero-grain emery paper, and coated with high-purity silver paste and then dried for $2 \mathrm{~h}$ at $150{ }^{\circ} \mathrm{C}$. Dielectric and impedance parameters were measured using an impedance analyzer (HIOKI LCR HI Tester, Model 3532) as a function of temperature over a wide range of frequencies $(100 \mathrm{~Hz}-5 \mathrm{MHz})$.

\section{Results and discussion}

\section{1 Structural study}

The differential thermal analysis (DTA) curve for MAS glass-ceramic is shown in Fig. 1. A broad endothermic peak in the DTA curve around $260{ }^{\circ} \mathrm{C}$ is due to the dehydration, reduction and some decomposition reactions taking place. The observed exothermic peak around $700{ }^{\circ} \mathrm{C}$ is indicative of the crystallization process. Bulk density of the sample measured at room temperature is found to be $1.72 \mathrm{~g} / \mathrm{ml}$.

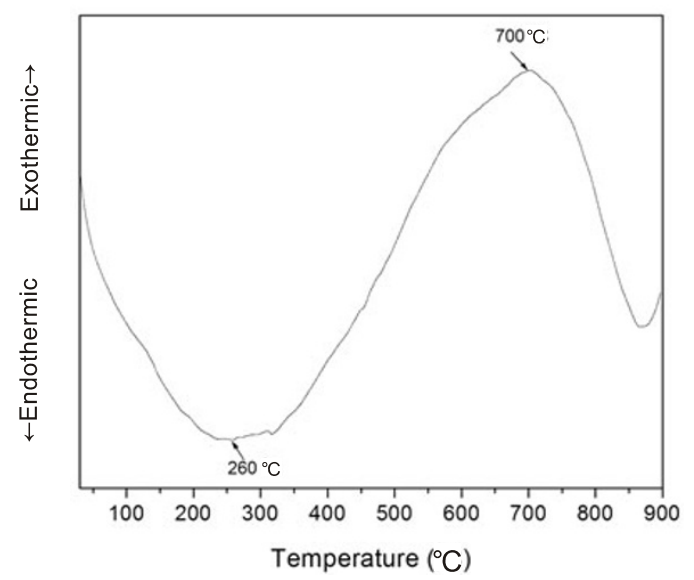

Fig. 1 DTA curve for MAS glass-ceramic prepared by sintering route.

The room-temperature XRD pattern of the sintered sample is shown in Fig. 2. The XRD analysis of the sample reveals that magnesium silicate fluoride (JCPDS 76-0638), cordierite (JCPDS 85-1722), together with minor traces of phlogopite (JCPDS 16-0352) and sapphirine (JCPDS 21-0549), were developed. The predominant crystalline phases are found to be magnesium silicate fluoride and cordierite.

SEM micrographs of the sample with different magnifications at room temperature are shown in Fig. 3. Figure 3(a) shows a highly densified microstructure with large amount of molten glass phases for MAS glass-ceramic. The photograph also shows the presence of plate-like microstructure (Fig. 3(b)). 


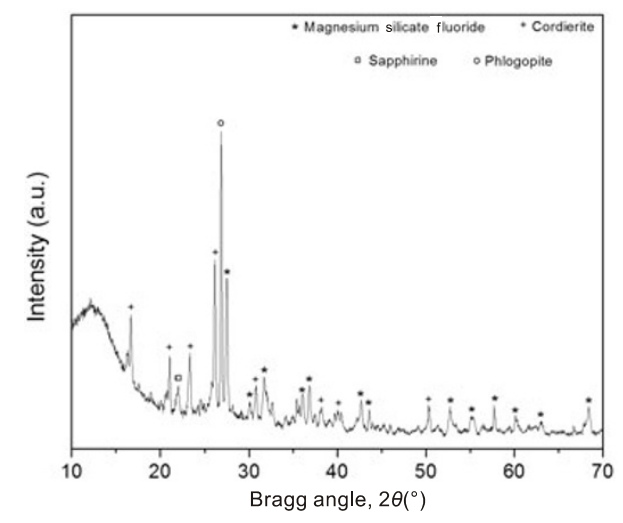

Fig. 2 Room-temperature XRD pattern of MAS glass-ceramic.

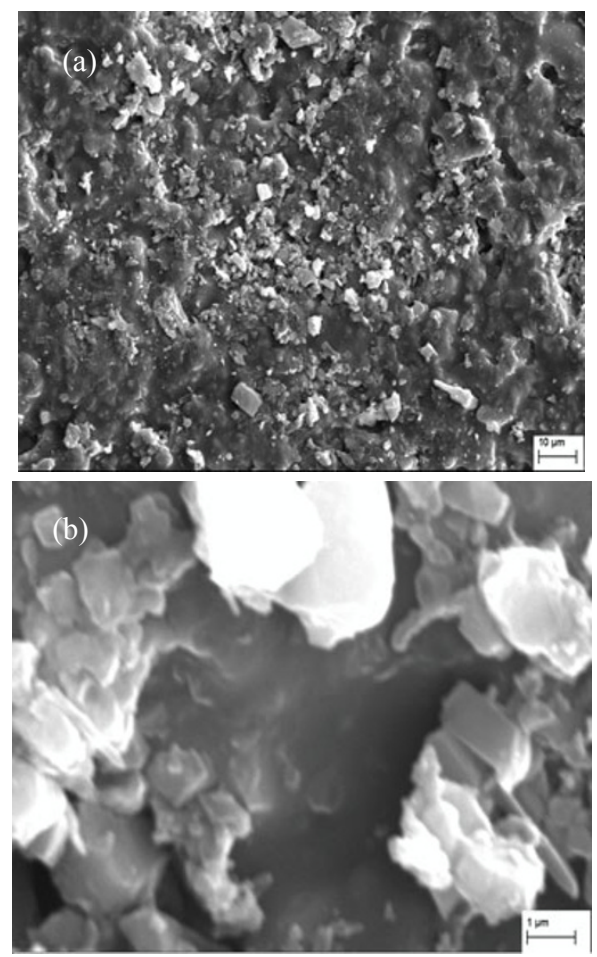

Fig. 3 SEM micrographs of fresh fractured surface of MAS glass-ceramic with different magnifications at room temperature.

\section{2 Dielectric properties}

Figure 4 shows the variation of relative dielectric constant $\left(\varepsilon_{\mathrm{r}}\right)$ and loss tangent $(\tan \delta)$ of MAS glass-ceramic with frequency at room temperature. Both the parameters decrease with the increasing frequency, indicating a normal behavior of dielectric materials having mobile charge carriers (i.e., ions and electrons) [7]. Table 1 shows the room-temperature $\varepsilon_{\mathrm{r}}$ and $\tan \delta$ values of the sample measured at different frequencies. The fall in dielectric constant arises from the fact that the polarization does not occur instantaneously with the application of the electric field as charges possess inertia. The delay in response towards the impressed alternating electric field leads to loss and hence decline in dielectric constant [8]. The loss tangent $(\tan \delta)$ also decreases with the increasing frequency. It is observed that at higher frequency, these parameters become almost frequency-independent. The dielectric constant and dielectric loss of the sample at $5 \mathrm{MHz}$ are 26.14 and 0.033 at room temperature, respectively.

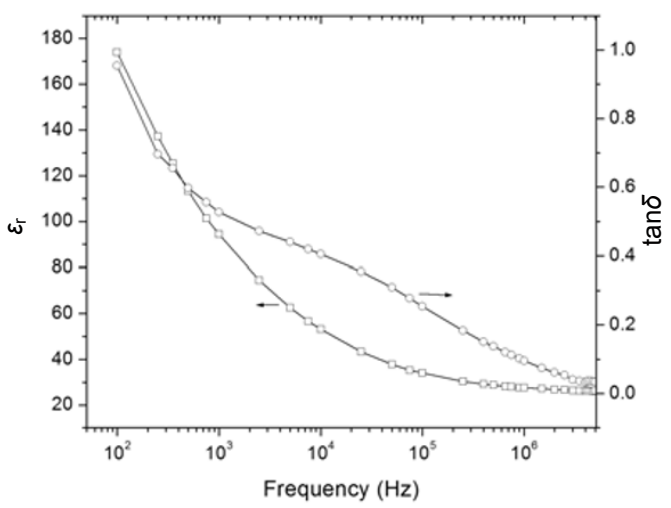

Fig. 4 Variation of dielectric constant $\left(\varepsilon_{\mathrm{r}}\right)$ and loss tangent $(\tan \delta)$ with frequency at room temperature.

Table 1 Physical relative properties of MAS glass-ceramic sintered at $1000{ }^{\circ} \mathrm{C}$

\begin{tabular}{ccccc}
\hline Physical parameters & \multicolumn{4}{c}{ Frequency } \\
\cline { 2 - 5 } (at room temperature) & $1 \mathrm{kHz}$ & $10 \mathrm{kHz}$ & $100 \mathrm{kHz}$ & $1 \mathrm{MHz}$ \\
\hline$\varepsilon_{\mathrm{r}}$ & 94.44 & 53.04 & 34.12 & 27.50 \\
$\tan \delta$ & 0.527 & 0.405 & 0.254 & 0.095 \\
\hline
\end{tabular}

The variation of relative dielectric constant $\left(\varepsilon_{\mathrm{r}}\right)$ with temperature at selected frequencies $(1 \mathrm{kHz}, 10 \mathrm{kHz}$, $100 \mathrm{kHz}$ and $1 \mathrm{MHz}$ ) is shown in Fig. 5(a). From these curves, it can be seen that dielectric constant increases almost linearly with the increasing temperature (i.e., $30-150{ }^{\circ} \mathrm{C}$ ) and then starts decreasing slowly. The broadening of dielectric peaks may be attributed to the disorder or defects presented in the system [8]. Since there is no anomaly at $150{ }^{\circ} \mathrm{C}$ in the DTA curve, the permittivity peak at $150{ }^{\circ} \mathrm{C}$ does not correspond to a phase transition. In general, a structural phasetransition temperature depends strongly on the chemical composition, and in this case, the anomaly was not observed in the cooling regime (not shown here) as it should be in a reversible process, so it is concluded that this experimental feature does not correspond to a structural phase transition. It is well accepted that the presence of $\mathrm{Mg}^{2+}$ cations could give 
rise to defects such as oxygen vacancies. The presence of $\mathrm{K}^{+}$cations must also be considered with the consequent generation of oxygen vacancies in the structure. All these features lead us to conclude that this anomaly is not intrinsic in character and may be induced by point defects. For higher temperatures $\left(>230{ }^{\circ} \mathrm{C}\right)$, the charge carriers are free to move through the crystal causing a polarization and hence $\varepsilon_{\mathrm{r}}$ increases. The increase in permittivity at higher temperatures is possibly due to the polarizability of $\mathrm{K}^{+}$ ions $\left(3.83 \AA^{3}\right)$, which is higher than that of $\mathrm{Mg}^{2+}$ ions $\left(1.32 \AA^{3}\right)$. Polycrystalline aggregates such as MAS glass-ceramics exhibit interfacial or space charge polarization arising from the differences amongst the conductivity of two phases (magnesium silicate fluoride and cordierite), which are present in the material. If phases of different conductivity present, the motion of charge carriers occurs readily through one phase, but it is interrupted when it reaches a phase boundary of other phases. This causes a build-up of charge at the interface, which corresponds to a large polarization and high value of effective dielectric constant. The charge accumulation at the grain
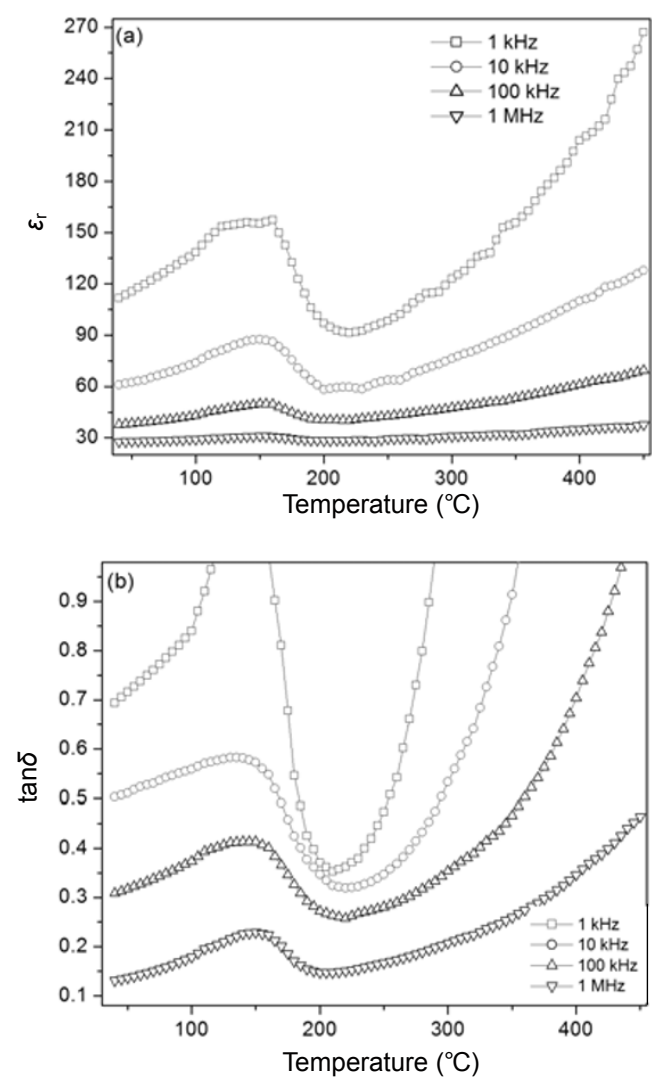

Fig. 5 Variation of (a) $\varepsilon_{\mathrm{r}}$ and (b) $\tan \delta$ with temperature for MAS glass-ceramic. boundaries is responsible for higher values of dielectric constant at low frequencies $[9,10]$. At high frequency, $\varepsilon_{\mathrm{r}}$ results from the grains which have a small dielectric constant [8]. It is interesting to note that as the temperature increases, the dielectric constant increases at all frequencies.

The variation of loss $\operatorname{tangent}(\tan \delta)$ with temperature for the sample is shown in Fig. 5(b). This increase in $\tan \delta$ may be due to the enhancement in conductivity of the material. The rapid increase of loss tangent at higher temperature in low frequency region may be due to space charge polarization. In addition, the high loss factor of MAS glass-ceramic at higher temperatures is due to the large glass content and the high mobility of alkali ions $\left(\mathrm{K}^{+}\right)$. It is observed that, even at high frequency $(1 \mathrm{MHz})$ and temperature $\left(450{ }^{\circ} \mathrm{C}\right)$, the dielectric $\operatorname{loss}(\tan \delta)$ remains relatively low (0.46), an important fact from the point of view of technological applications.

\section{3 Conductivity study}

Figure 6 shows the variation of AC conductivity $\left(\ln \sigma_{\mathrm{AC}}\right)$ as a function of the inverse of absolute temperature $\left(10^{3} / T\right)$ at four different frequencies. The AC conductivity $\left(\sigma_{\mathrm{AC}}\right)$ and activation energy $\left(E_{\mathrm{a}}\right)$ of the sample was calculated using the relation $\sigma=\omega \varepsilon_{\mathrm{r}} \varepsilon_{0} \tan \delta$ and $\sigma=\sigma_{0} \exp \left(-E_{\mathrm{a}} / k_{\mathrm{B}} T\right)$, where $\varepsilon_{0}$ is the vacuum permittivity; $\omega$ is the angular frequency; $k_{\mathrm{B}}$ is Boltzmann constant, and $\sigma_{0}$ is the pre-exponential factor. Conductivity of the compound at higher temperature is higher, which is a very common behavior in most of dielectric ceramics [11]. Because alkali metal ions (such as $\mathrm{Na}^{+}, \mathrm{Li}^{+}$and $\mathrm{K}^{+}$) are source of conductive carriers in glass-ceramic, the composition and content are closely related with the electrical conductivity of glass-ceramic. In this ceramic, $\mathrm{K}^{+}$ions are mobile and contribute to the conductivity. The higher values of conductivity at higher temperatures are possibly due to the movement of mobile $\mathrm{K}^{+}$and $\mathrm{F}^{-}$ions. In the lower temperature region (Region I), there is linear variation in conductivity with temperature with strong frequency dependence. The region which lies in the temperature range from $150{ }^{\circ} \mathrm{C}$ to around $230{ }^{\circ} \mathrm{C}$ does not obey the conductivity relation and its trend seems as a metallic behavior. In the higher temperature range (Region II), conductivity varies exponentially with temperature but with relatively weak frequency dependence. The nature of variation of $\sigma_{\mathrm{AC}}$ over a wide temperature range supports the thermally activated transport properties of 
the material. The activation energy $\left(E_{\mathrm{a}}\right)$ of the compound was calculated from the slope of $\ln \sigma$ vs. 1/T curves. The values of $E_{\mathrm{a}}$ at different frequencies are given in Table 2.

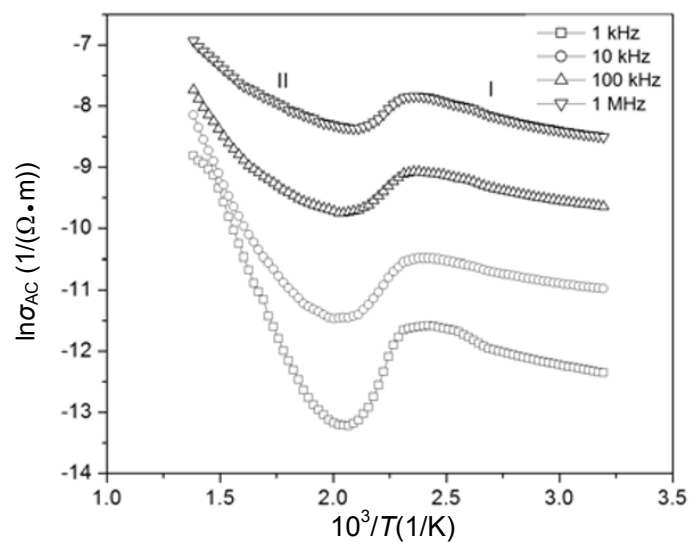

Fig. 6 Variation of AC conductivity $\left(\ln \sigma_{\mathrm{AC}}\right)$ as a function of the inverse of absolute temperature $\left(10^{3} / T\right)$ at four different frequencies for MAS glass-ceramic.

Table 2 Activation energy of MAS glassceramic sintered at $1000{ }^{\circ} \mathrm{C}$

\begin{tabular}{ccc}
\hline Activation energy & \multicolumn{2}{c}{ Temperature range } \\
\cline { 2 - 3 }$E_{\mathrm{a}}(\mathrm{eV})$ & Region I & Region II \\
\hline at $1 \mathrm{kHz}$ & 0.09 & 0.76 \\
at $10 \mathrm{kHz}$ & 0.06 & 0.51 \\
at $100 \mathrm{kHz}$ & 0.06 & 0.30 \\
at $1 \mathrm{MHz}$ & 0.07 & 0.19 \\
\hline
\end{tabular}

The activation energy is found to increase with increasing temperature. This behavior suggests that the conduction mechanism in MAS glass-ceramic may be due to the hopping of charge carriers between different localized states. The value of activation energy in Region I is found to be very low. The small values of activation energy in Region II may be due to the singly ionized oxygen vacancies. Therefore, the conductivity may be of mixed type (i.e., ionic-polaronic and space charge) $[12,13]$.

\section{4 Impedance analysis}

The electrical behavior of the system was studied over a wide range of frequency and temperature using a complex impedance spectroscopy (CIS) technique. This technique enables us to separate the real and imaginary components of the complex impedance parameters, and hence provides information of the structure-property relationship of the sample. A polycrystalline material usually has grain and grain boundary properties with different time constants at high temperature leading to two successive semicircles. It can conventionally be displayed in a complex plane plot (Nyquist diagram) in terms of the following formalism:

$$
\text { Complex impedance } \quad Z^{*}(\omega)=Z^{\prime}-\mathrm{j} Z^{\prime \prime}
$$

where $Z^{\prime}=|Z| \cos \theta$ and $Z^{\prime \prime}=|Z| \sin \theta$.

Figure 7(a) shows a set of Nyquist plots $\left(Z^{\prime \prime}\right.$ vs. $\left.Z^{\prime}\right)$ over a wide range of frequency $(100 \mathrm{~Hz}-5 \mathrm{MHz})$ at different temperatures. The effect of temperature on impedance parameter of the material becomes clearly visible with the rise in temperature. A single semicircular arc has been observed in a wide temperature range $\left(\leqslant 400{ }^{\circ} \mathrm{C}\right)$. This indicates that the electrical properties of the material arise mainly due to the bulk effects. As temperature increases, the intercept point on the real axis shifts towards the origin,
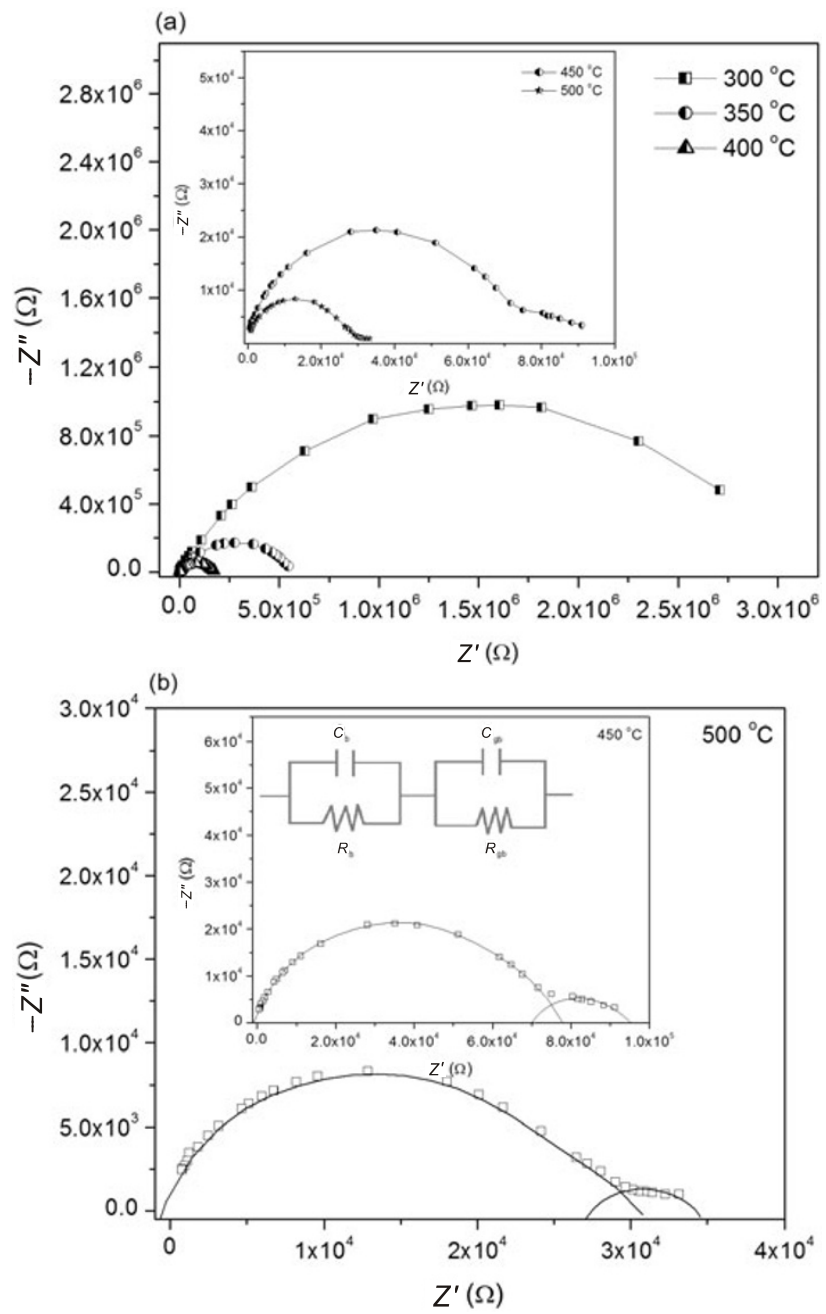

Fig. 7 (a) Nyquist plots of MAS glass-ceramic at different temperatures; (b) Nyquist plots of MAS glass-ceramic with equivalent circuit (inset). 
indicating the decrease in the resistive property of the material. The intercept of the semicircle on the real axis is the bulk resistance $\left(R_{\mathrm{b}}\right)$ of the sample. The values of bulk resistance $\left(R_{\mathrm{b}}\right)$ and bulk capacitance $\left(C_{\mathrm{b}}\right)$ are shown in Table 3. This type of electrical behavior can be explained in terms of an equivalent circuit comprising of a parallel combination of $R C$ circuits. At much higher temperatures $\left(\geqslant 450{ }^{\circ} \mathrm{C}\right)$, it was possible to trace two semicircles (Fig. 7(b)). The appearance of two semicircles suggests the presence of both bulk as well as grain boundary effects in the polycrystalline sample. Each semicircle is a representative of an $R C$ circuit that corresponds to individual component of the material (Fig. 7(b) inset). The presence of pores modifies the complex impedance plots by changing the diameters of the grain interior or grain boundary arc without introducing a new arc or other characteristic in the impedance spectrum. Thus, it is not possible to quantify the degree or nature of porosity using impedance spectrum.

Table 3 Values of bulk resistance $\left(R_{\mathrm{b}}\right)$, relaxation frequency $\left(f_{\mathrm{r}}\right)$, and bulk capacitance $\left(C_{\mathrm{b}}\right)$ of MAS glass-ceramic at different temperatures

\begin{tabular}{cccc}
\hline$T\left({ }^{\circ} \mathrm{C}\right)$ & $R_{\mathrm{b}}(\mathrm{k} \Omega)$ & $f_{\mathrm{r}}(\mathrm{kHz})$ & $C_{\mathrm{b}}(\mathrm{pF})$ \\
\hline 300 & 3059.17 & 0.65 & 80 \\
350 & 569.38 & 5 & 56 \\
400 & 165.25 & 25 & 38 \\
450 & 75.07 & 75 & 28 \\
500 & 27.19 & 250 & 23 \\
\hline
\end{tabular}

Figure 8 exhibits the variation of the real part of impedance ( $\left.Z^{\prime}\right)$ with frequency at different temperatures. The decrease in the magnitude of $Z^{\prime}$ with increase in both frequency as well as temperature indicates the increase in $\mathrm{AC}$ conductivity. The values of $Z^{\prime}$ merge at higher frequencies $(\geqslant 100 \mathrm{kHz})$, which indicates the release of space charges. Figure 9 represents the impedance loss spectra (i.e., the variation of the imaginary part of impedance $\left(Z^{\prime \prime}\right)$ with frequency). The curves show that the value of $Z^{\prime \prime}$ reaches maximum value, $Z_{\max }^{\prime \prime}$, at all temperatures. The average peak position regularly changes towards the higher frequency side on increasing temperature. Furthermore, as the temperature increases, the magnitude of $Z^{\prime \prime}$ decreases and the effect is more pronounced at the peak position. The shift of the peaks towards higher frequency on increasing temperature is due to the reduction in the bulk resistivity. The relaxation time $(\tau)$ was calculated from the frequency maxima $\left(f_{\max }\right)$ at
$Z_{\max }^{\prime \prime}$. At the peak, the relaxation is defined by the condition:

$$
\omega_{\max } \tau=2 \pi f_{\max } \tau=1
$$

where $\tau$ is the relaxation time at $f_{\max }$ and $f_{\max }$ is the relaxation frequency. It is independent of the sample's geometrical factors and depends basically on the intrinsic properties (i.e., microstructure) of the material only. The $\tau$ value is found to be decreasing on increasing temperature, which is a typical semiconductor behavior. The nature of variation of $\tau$ with temperature shows that the relaxation process is temperature dependant. The variation of $\tau$ as a function of the inverse of absolute temperature is shown in Fig. 10.

The activation energy evaluated from the slope of the curve, using the relation $\tau=\tau_{0} \exp \left(-E_{\mathrm{a}} / k_{\mathrm{B}} T\right)$ (where $\tau_{0}$ is the pre-exponential factor; $E_{\mathrm{a}}$ is the activation energy; $k$ is Boltzmann constant, and $T$ is absolute temperature), is found to be $0.48 \mathrm{eV}$.

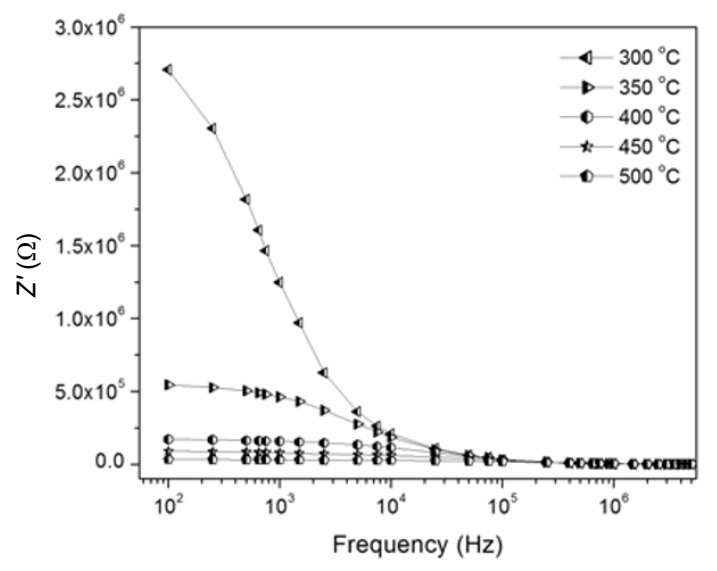

Fig. 8 Variation of the real part of impedance $\left(Z^{\prime}\right)$ of MAS glass-ceramic as a function of frequency.

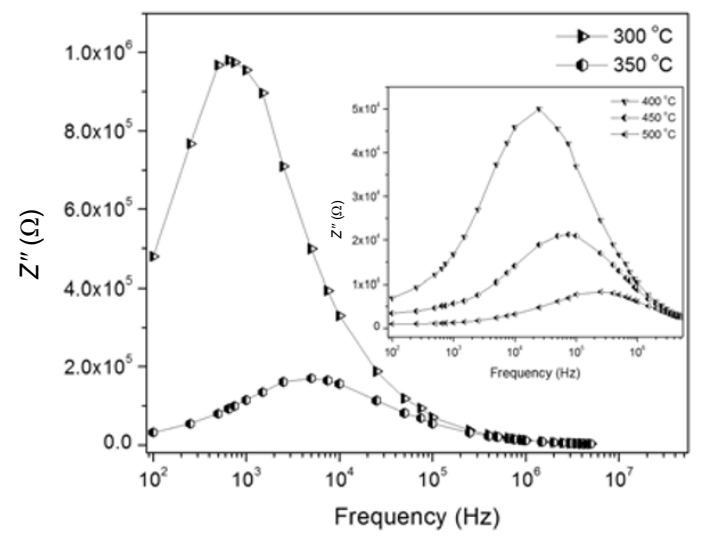

Fig. 9 Variation of the imaginary part of impedance $\left(Z^{\prime \prime}\right)$ of MAS glass-ceramic as a function of frequency. 


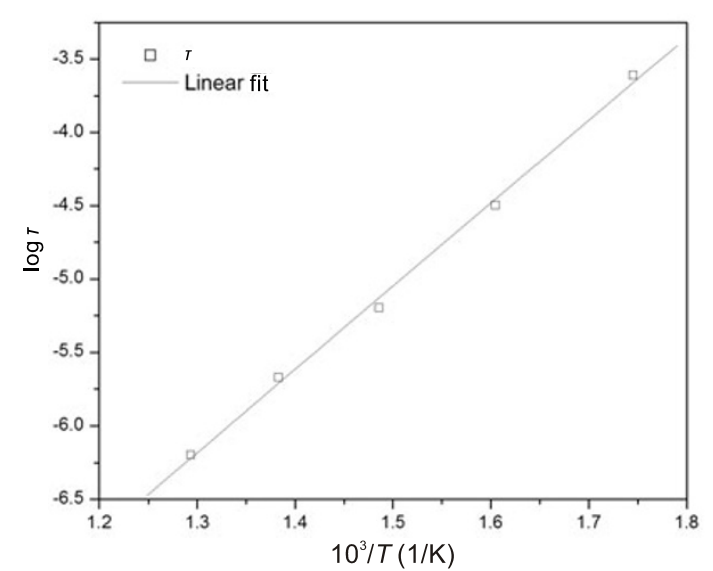

Fig. 10 Variation of the relaxation time $(\tau)$ as a function of temperature.

\section{5 Modulus studies}

Impedance spectroscopy is a powerful technique for characterization of grain boundaries in ceramic materials. The advantage of adopting complex electric modulus formalism is that it can discriminate electrode polarization from grain boundary conduction process. In polycrystalline materials, impedance formalism might emphasize grain boundary conduction process, while bulk effects on frequency domain would dominate in the electric modulus formalism. The use of modulus spectroscopy plot is particularly useful for separating components with similar resistance but different capacitance. The other advantage of electric modulus formalism is that the electrode effect is suppressed. Due to the above reasons, the complex electric modulus $\left(M^{*}\right)$ have been calculated from the impedance data using the following relation:

$$
\begin{gathered}
M^{*}(\omega)=1 / \varepsilon(\omega)=M^{\prime}+\mathrm{j} M^{\prime \prime} \\
M^{\prime}=\omega C_{0} Z^{\prime \prime} \text { and } M^{\prime \prime}=\omega C_{0} Z^{\prime}
\end{gathered}
$$

where $\omega$ is the angular frequency $(2 \pi f) ; C_{0}=\varepsilon_{0}(A / t)$ is the geometrical capacitance; $\varepsilon_{0}$ is the permittivity of free space; $A$ is the area of the electroded surface and $t$ is the thickness.

Figure 11(a) shows the variation of $M^{\prime}$ with frequency for MAS glass-ceramic at selected temperatures. A very low value ( $\sim$ zero) of $M^{\prime}$ in the low frequency region is observed. Continuous increases in the dispersion on increasing frequency for all the temperatures are observed. Such observations could possibly be related to a lack of restoring force governing the mobility of the charge carriers under the action of an induced electric field. This confirms the elimination of electrode effect in the material. The value of $M^{\prime}$ decreases with the rise in temperature in the high frequency region. Figure 11(b) shows the variation of $M^{\prime \prime}$ with frequency over a wide temperature range and frequencies ascribing correlation between motions of mobile ions [14]. A well-defined relaxation mechanism is observed in temperature range $300-500{ }^{\circ} \mathrm{C}$. The relaxation peaks shift towards higher frequency side with the rise in temperature. At frequencies lying to the left of the peaks, the charge carriers $\left(\mathrm{K}^{+}\right.$and $\mathrm{F}^{-}$ions) may be considered as mobile over long distances whereas in the region to the right of the peaks, charge carrier motion is generally confined in their potential well. The nature of modulus spectrum suggests the existence of hopping mechanism of electrical conduction in the material. The value of $M^{\prime \prime}$ increases as a function of temperature.
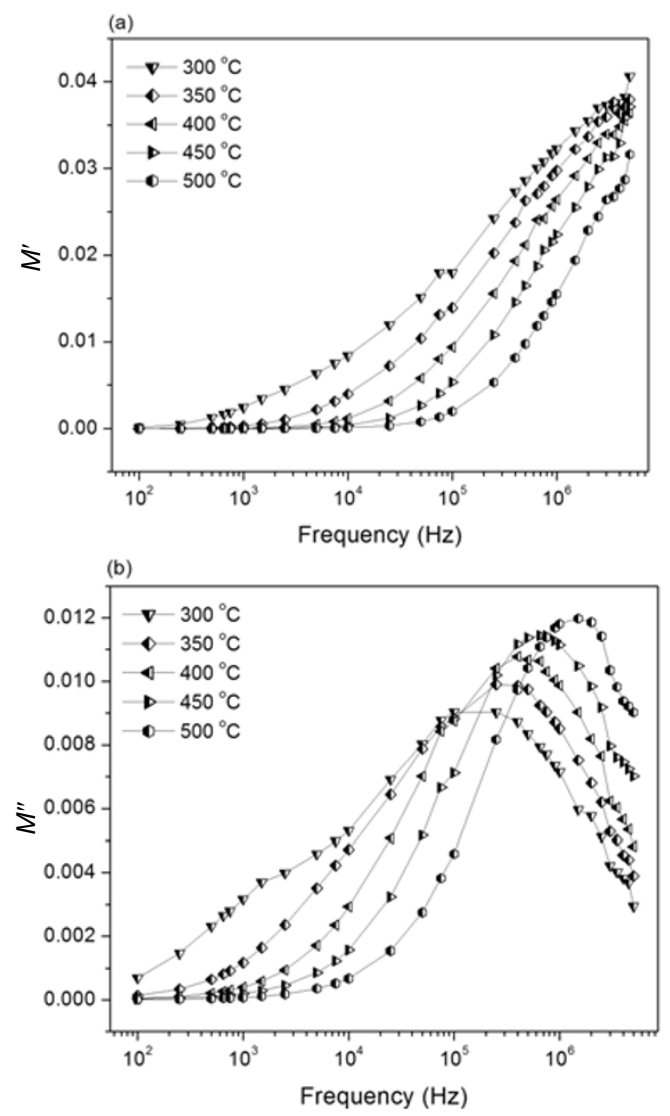

Fig. 11 Variation of (a) $M^{\prime}$ and (b) $M^{\prime \prime}$ with frequency of MAS glass-ceramic at selected temperatures.

\section{Conclusions}

MAS sample was prepared via sintering route. XRD 
analysis reveals several crystalline phases formed in this material: magnesium silicate fluoride, cordierite, together with minor traces of phlogopite and sapphirine. The predominant crystalline phases are found to be magnesium silicate fluoride and cordierite. Dielectric constant and dissipation factor decrease with the increase in frequency. The increase of $\varepsilon_{\mathrm{r}}$ at higher temperatures may be due to the weakening of binding force between the ions leading to mobile ions $\left(\mathrm{K}^{+}\right.$and $\mathrm{F}^{-}$) contribution. CIS enables us to separate the grain and grain boundary contributions in the material. Both the grain and grain boundary resistances decrease with the rise in temperature. We used an equivalent circuit to explain electrical phenomena occurring inside the material. The nature of variation of AC conductivity with temperature exhibits NTCR behavior of the sample like that of a semiconductor. The activation energies at higher temperatures are found to be less than $1 \mathrm{eV}$. This suggests that polarons at lower temperatures, singly ionized oxygen vacancies and long range conductivity associated with extrinsic mechanisms due to the presence of $\mathrm{K}^{+}$and $\mathrm{F}^{-}$ions at higher temperatures contribute to the conduction process in the material.

Open Access: This article is distributed under the terms of the Creative Commons Attribution Noncommercial License which permits any noncommercial use, distribution, and reproduction in any medium, provided the original author(s) and source are credited.

\section{References}

[1] Grossam DG. Machinable glass-ceramics based on tetrasilicic mica. J Am Ceram Soc 1972, 55: 446-449.

[2] Baik DS, No KS, Chun JS, et al. A comparative evaluation method of machinability for mica-based glass-ceramics. J Mater Sci 1995, 30: 1801-1806.

[3] Boccaccini AR. Machinability and brittleness of flass-ceramics. J Mater Process Tech 1997, 65: 302-304.

[4] Pannhorst W. Glass ceramics: State-of-the-art. J Non-Cryst Solids 1997, 219: 198-204.

[5] Xu HHK, Jahanmir S. Scratching and grinding of a machinable glass-ceramic with weak interfaces and rising T-curve. J Am Ceram Soc 1995, 78: 497-500.

[6] Goswami M, Mirza T, Sarkar A, et al. Preparation and characterization of magnesium-aluminium-silicate glass ceramics. Bull Mater Sci 2000, 23: 377-382.

[7] Anderson JC. Dielectrics. London: Chapman \& Hall, 1964.

[8] Prasad A, Basu A. Structural and dielectric studies of $\mathrm{Li}_{2} \mathrm{SiO}_{3}$ ceramic. Mater Lett 2012, 66: 1-3.

[9] Jawahar K, Choudhary RNP. Structural, thermal and dielectric properties of $\mathrm{La}_{3 / 2} \mathrm{Bi}_{3 / 2} \mathrm{Fe}_{5} \mathrm{O}_{12}$. Solid State Commun 2007, 142: 449-452.

[10] Hsiao YJ, Chang YH, Fang TH, et al. Dielectric relaxation properties of perovskite-pyrochlore biphase ceramics. Appl Phys Lett 2005, 87: 142906.

[11] Rao PSVS, Rao KS, Bhanumathi A, et al. Anomalous electrical behaviour of barium sodium niobate ceramics doped with trivalent lanthanum. J Mater Sci Lett 1987, 6: 809-810.

[12] Deng GC, Li GR, Ding AL, et al. Evidence for oxygen vacancy inducing spontaneous normalrelaxor transition in complex perovskite ferroelectrics. Appl Phys Lett 2005, 87: 192905.

[13] Molak A, Talik E, Kruczek M, et al. Characterisation of $\mathrm{Pb}\left(\mathrm{Mn}_{1 / 3} \mathrm{Nb}_{2 / 3}\right) \mathrm{O}_{3}$ ceramics by SEM, XRD, XPS and dielectric permittivity test. Mat Sci Eng B 2006, 128: $16-24$.

[14] Borsa F, Torgeson DR, Martin SW, et al. Relaxation and fluctuations in glassy fast-ion conductors: Wide-frequency-range NMR and conductivity measurements. Phys Rev B 1992, 46: 795-800. 